\title{
Delayed extensive right brachial and radial artery thrombosis: A rare complication of transradial cardiac catheterization
}

\author{
Kashyap Shah ${ }^{*}$, Abdelsalam Elshaikh ${ }^{1}$, Matthew Krinock ${ }^{1}$ and Cara Ruggeri ${ }^{1,2}$ \\ ${ }^{1}$ Department of Internal Medicine, St. Luke's University Hospital, Bethlehem, PA, USA \\ ${ }^{2}$ Clinical Associate Professor of Medicine, Lewis Katz School of Medicine, Temple University School of Medicine, Philadelphia, PA, USA
}

\begin{abstract}
Cardiac catheterization via transradial approach (TRA) has increased in prevalence surpassing femoral artery approach. Radial artery occlusion (RAO), a well-known complication post cardiac catheterization, typically occurs immediately after cardiac catheterization and is usually asymptomatic not requiring any intervention. We encounter a unique case of extensive brachial and radial artery occlusion more than two months after cardiac catheterization, demonstrating the importance of a thorough history, and the need for increased awareness regarding the possibility of delayed presentation of cardiac catheterization complications.
\end{abstract}

\section{Introduction}

Radial artery occlusion (RAO) is a common complication after cardiac catheterization with transradial approach (TRA). However, it is usually reported within days following catheterization, and infrequently requires intervention [1]. We encounter a unique case of extensive distal brachial and radial arterial thrombosis two months after cardiac catheterization with transradial approach.

\section{Case report}

A 75-year-old female presents to the emergency room with acute onset of right upper extremity pain worse with movement, associated with paresthesia, pallor and discoloration of her right fingers for 4 days (Figures 1a and 1b). Her past medical history was significant for non-obstructive coronary artery disease, hypertension, hyperlipidemia, hypothyroidism, remote history of colon cancer successfully treated with surgical resection and Raynaud's disease. The patient also reported to have recent history of cardiac catheterization with transradial approach over two months ago. Physical exam was significant for nonpalpable right radial pulse as well as distal cyanosis and cool fingers. Right upper extremity arterial duplex showed acute thrombus in the

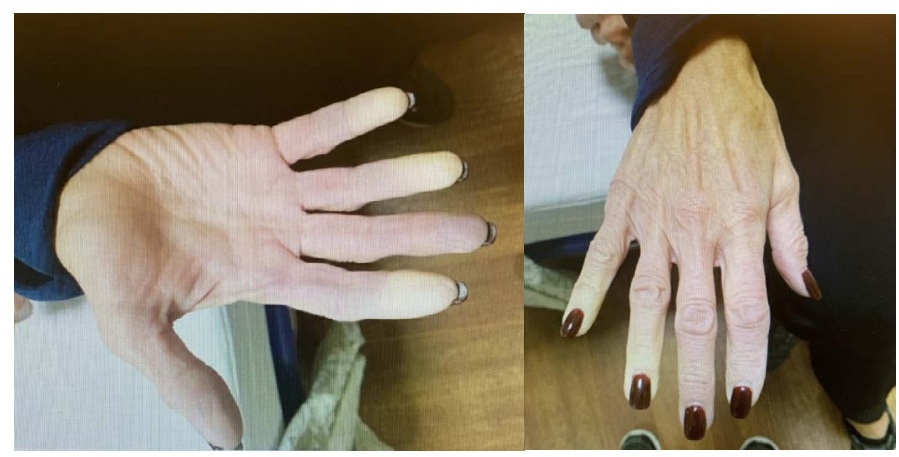

Figure 1a and 1b. Showing pallor of right hand due to brachial and radial artery occlusion. (images reproduced with patient permission) distal brachial artery below the antecubital fossa, extending into the radial artery from the proximal forearm to the wrist. Right ulnar artery was patent. The patient was immediately started on heparin drip and was taken to the operating room for successful emergent open thromboembolectomy. The patient was restarted on heparin after the surgery and eventually bridged to Warfarin. The patient had complete resolution of her claudication symptoms with palpable right brachial and radial pulses. Hypercoagulable work up and age appropriate cancer screening including recent colonoscopy, mammogram and CT abdomen/pelvis (performed due to remote history of colon cancer) were unremarkable.

\section{Discussion}

Our patient had extensive brachial and radial artery occlusion (RAO) as a complication of her cardiac catheterization. RAO is the most common complication of transradial cardiac catheterization, reported in $1-10 \%$ of cases [2]. Transradial approach is increasingly favoured due to patient satisfaction, early ambulation, decreased length of stay and low risk of major bleeding [3]. The pathogenesis of RAO is believed to be due to endothelial injury and decreased radial flow after sheath and catheter insertion. Furthermore, repeated radial artery cannulation can promote intimal hyperplasia resulting in negative remodeling of arterial wall and further predisposition to RAO. Most cases of RAO are asymptomatic due to a dual blood supply to the hand from ulnar artery and rich collateral circulation. According to a prospective study of 1600 patients who underwent cardiac catheterization with transradial approach, the incidence rate of major vascular complication including

${ }^{\star}$ Correspondence to: Kashyap Shah, Department of Internal Medicine, St. Luke's University Hospital, 801 Ostrum Street, Fountain Hill, Bethlehem, PA 18015, USA, Tel: +1 215-353-7599, E-mail: kashyap.shah@sluhn.org

Key words: cardiac catheterization complications, transradial approach (TRA), radial artery occlusion (RAO)

Received: March 23, 2020; Accepted: April 06, 2020; Published: April 15, 2020 
limb ischemia was only $0.44 \%$ [4]. In addition, the incidence of RAO decreases with time (incidence of $7.7 \%$ within 24 hours vs $5.5 \%$ at $>1$ week follow up) [1]. RAO is an important complication as it can prevent subsequent use of occluded artery for any future cardiac catheterizations. In our case, the combination of both the extensive disease as well as the late presentation brings awareness to the potential devastating long term complications of radial artery catheterization, and also highlights the need for a thorough history despite possible remote procedures for patients presenting with evidence of thrombosis. Fortunately, our patient had a good outcome with timely surgical intervention.

\section{Conclusion}

This case highlights the importance of thorough history and brings awareness to the possibility of devastating delayed complications of cardiac catheterization with transradial approach.

\section{Conflict of interest}

The authors declare that there is no conflict of interest.

\section{References}

1. Rashid M, Kwok CS, Pancholy S, Chugh S, Kedev SA, et al. (2016) Radial artery occlusion after transradial interventions: A systematic review and meta-analysis. $J \mathrm{Am}$ Heart Assoc 5: 1-22. [Crossref]

2. Avdikos G, Karatasakis A, Tsoumeleas A, Lazaris E, Ziakas A, et al. (2017) Radial artery occlusion after transradial coronary catheterization. Cardiovasc Diagn Ther 7 305-316. [Crossref]

3. Sachdeva S, Saha S (2014) Transradial approach to cardiovascular interventions: An update. Int J Angiol 23: 77-84. [Crossref]

4. Hahalis G, Tsigkas G, Kakkos S, Panagopoulos A, Tsota I et al. (2016) Vascular Complications Following Transradial and Transulnar Coronary Angiography in 1600 Consecutive Patients. Angiology 67: 438-443. [Crossref]

Copyright: C2020 Shah K. This is an open-access article distributed under the terms of the Creative Commons Attribution License, which permits unrestricted use, distribution, and reproduction in any medium, provided the original author and source are credited. 